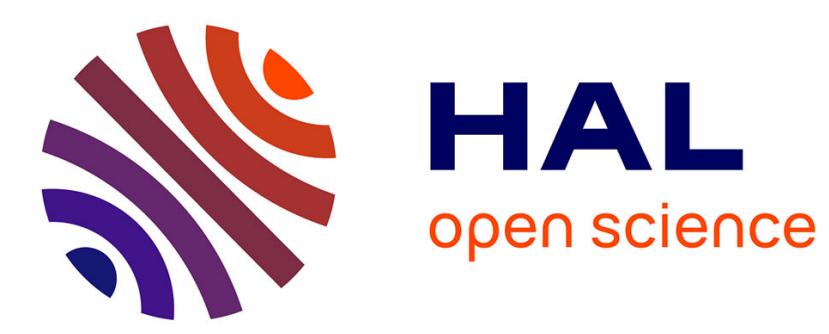

\title{
Loss-tolerant parity measurement for distant quantum bits
}

\author{
Alain Sarlette, Mazyar Mirrahimi
}

\section{To cite this version:}

Alain Sarlette, Mazyar Mirrahimi. Loss-tolerant parity measurement for distant quantum bits. Physical Review A: Atomic, molecular, and optical physics [1990-2015], 2017, 95 (3), 10.1103/PhysRevA.95.032329 . hal-01395590v2

\section{HAL Id: hal-01395590 https://inria.hal.science/hal-01395590v2}

Submitted on 14 Nov 2017

HAL is a multi-disciplinary open access archive for the deposit and dissemination of scientific research documents, whether they are published or not. The documents may come from teaching and research institutions in France or abroad, or from public or private research centers.
L'archive ouverte pluridisciplinaire HAL, est destinée au dépôt et à la diffusion de documents scientifiques de niveau recherche, publiés ou non, émanant des établissements d'enseignement et de recherche français ou étrangers, des laboratoires publics ou privés. 


\title{
Loss-tolerant parity measurement for distant quantum bits
}

\author{
Alain Sarlette ${ }^{1,2}$ and Mazyar Mirrahimi ${ }^{1,3}$ \\ ${ }^{1}$ QUANTIC project-team, INRIA Paris, France \\ ${ }^{2}$ Department of Electronics and Information Systems, Ghent University, Belgium \\ ${ }^{3}$ Yale Quantum Institute, Yale University, USA \\ (Received 20 April 2016; published 27 March 2017)
}

\begin{abstract}
We propose a scheme to measure the parity of two distant qubits, while ensuring that losses on the quantum channel between them does not destroy coherences within the parity subspaces. This capability enables deterministic preparation of highly entangled qubit states whose fidelity is not limited by the transmission loss. The key observation is that, for a probe electromagnetic field in a particular quantum state; namely, a superposition of two coherent states of opposite phases, the transmission loss stochastically applies a near-unitary backaction on the probe state. This leads to a parity measurement protocol where the main effect of the transmission losses is a decrease in the measurement strength. By repeating the nondestructive (weak) parity measurement, one achieves a high-fidelity entanglement in spite of a significant transmission loss.
\end{abstract}

DOI: 10.1103/PhysRevA.95.032329

\section{INTRODUCTION}

The correlation of distant systems through entanglement is a hallmark of quantum physics [1,2] and plays a fundamental role in envisioned quantum technology. Most fundamentally, quantum teleportation [3] shows how entanglement is a resource for effectively transmitting the unknown state of a quantum system, without physically transmitting quantum states. Towards future quantum computers, such teleportation could transport information between few-qubit processing units and memory units which must be well isolated and hence not directly coupled to the rest of the system via tunable physical interactions. This so-called modular architecture for quantum computing provides a viable solution to the major scaling problem for many-qubit quantum information processing [4,5]. In quantum communication, similar ideas would allow quantum repeaters to purify information through local operations only, provided they can consume units of entanglement between the communicating devices [6,7]; such quantum repeaters are a necessary technology for accurate quantum communication, with associated, e.g., cryptographic benefits, over long distances.

A major challenge towards enabling these applications is that generating entangled states between distant systems must rely itself on a quantum channel (see impossibility with local operations and classical communication). Microwave experiments have demonstrated how to deterministically entangle separate quantum subsystems via parity measurements [8], yet with a fidelity directly limited by the quality of the quantum channel: any losses on the probe field imply losses in entanglement. Channel losses can also be made to affect preparation success probability, instead of preparation fidelity. Many theoretical proposals [9-13] and experiments [14-19] have considered high-fidelity entanglement generation, despite propagation losses, by heralding the preparation on some rare photodetection events. The success rate of this probabilistic preparation is, however, very low in current experiments, while the preparation fidelity is still limited by imperfections such as dark counts of the photodetector. Recent developments in quantum superconducting circuit setups, like Refs. [20-25], show that local operations are becoming extremely reliable and fast, e.g., manipulating "cat states" as efficiently as single photons and having continuous field measurements instead of photon counters. The remaining bottleneck towards entanglement stabilization is then the channel loss.

This paper provides an explicit proposal for solving this problem through the achievement of loss-tolerant degeneracypreserving quantum nondemolition (QND) parity measurements between spatially separated qubits. Our key idea is to transmit over the quantum channel particularly engineered quantum states of light, i.e., "cat states", for which the dominant photon loss errors almost reduce to photon-number parity flips [26]. With this we design the interaction between qubits and probe field such that (i) measuring the probe at the output performs a QND measurement of qubit parity and (ii) photon loss events on the transmitted probe field render the detection less decisive (weak measurement) but, to a very good approximation, do not break the degeneracy of the parity subspaces. This measurement enables an efficient entanglement stabilization scheme, which not only generates highly entangled states but also protects them over arbitrarily long times against various noise channels.

The rather standard abstract setting [Fig. 1(a)] comprises two target qubits $\left|q_{A}\right\rangle,\left|q_{B}\right\rangle$ at different locations $\mathrm{A}, \mathrm{B}$ and possibly embedded in auxiliary quantum machinery, e.g., a cavity in circuit quantum electrodynamics (QED) setups [27]. For each measurement, a source generates a controlled "probe" quantum state $\left|\psi_{p}\right\rangle$ at A which then interacts with $\left|q_{A}\right\rangle$ according to a unitary $U_{A}$, is transmitted over a noisy quantum channel $C$, interacts with $\left|q_{B}\right\rangle$ according to $U_{B}$, and finally hits a detector at $\mathrm{B}$. Those probe states play the role of parity meter. Since the quantum channel is the unequivocal bottleneck for remote entanglement in state-of-the-art technology [28], we focus on this issue and assume in this paper that the local actions (i.e., $U_{A}, U_{B}$, generating $\left|\psi_{p}\right\rangle$, detection at $B$ ) are implemented perfectly. As we will show later, imperfections on such operations will have a small effect on the steady state when we stabilize quantum entanglement via feedback.

\section{DEGENERACY-PRESERVING PARITY MEASUREMENT}

The QND measurement of a quantum observable $Q$ discriminates possibly imperfectly between the eigenspaces of 


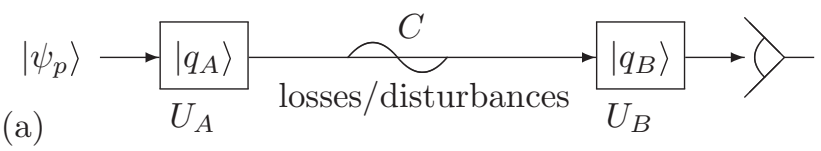

(b)
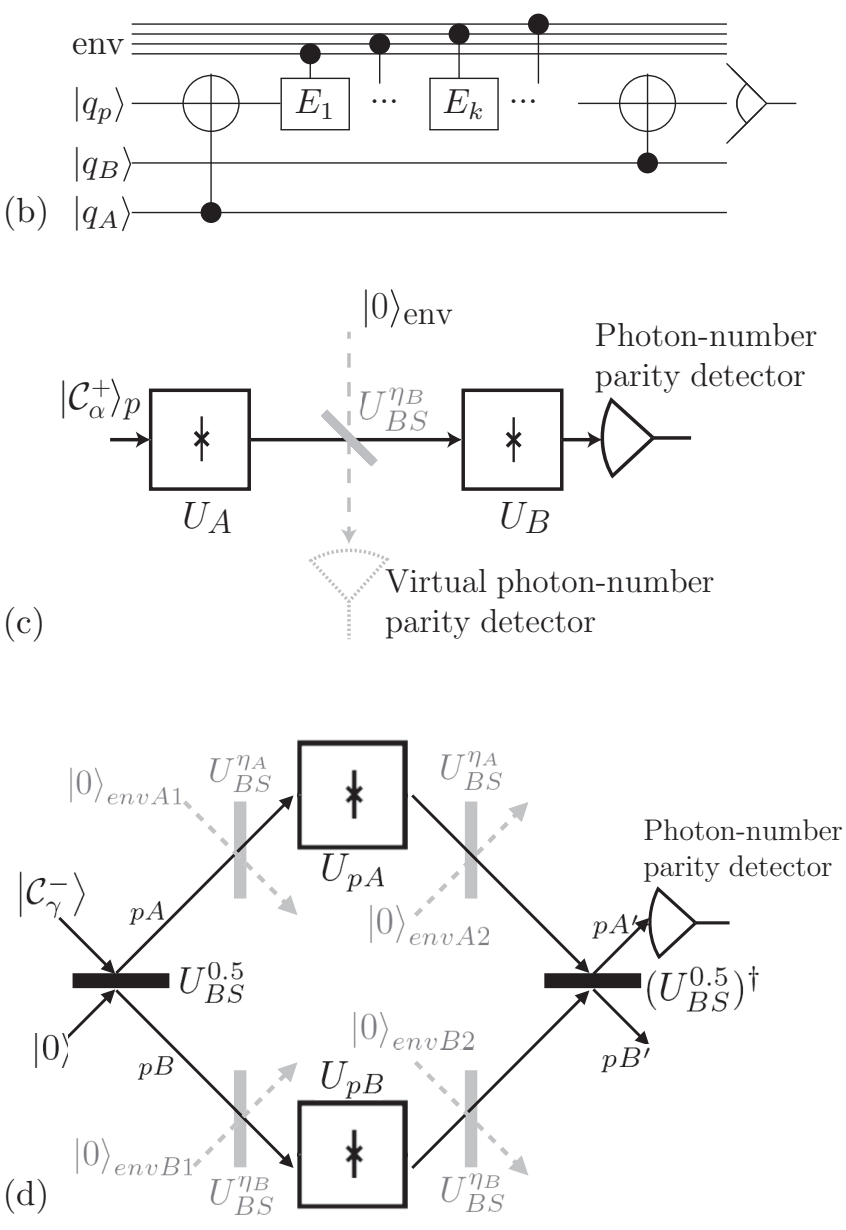

FIG. 1. (a) General setup for remote parity measurement with a probe $\left|\psi_{p}\right\rangle$ propagating on a noisy quantum channel $C$ between two target qubits $\left|q_{A}\right\rangle$ and $\left|q_{B}\right\rangle$. (b) Quantum logic circuit summarizing our concept with CNOT gates involving target qubits $\left|q_{A}\right\rangle,\left|q_{B}\right\rangle$ and a probe qubit $\left|q_{p}\right\rangle$. We represent the unknown corrupting operations on the quantum channel as applying known $E_{k}, k=1,2, \ldots$ conditionally on unknown states of the environment (env). (c) Corresponding experimental setup with a probe field initialized in a coherent superposition of two opposite coherent states ("cat state"). (d) Alternative concurrent scheme with entangled probe fields between two 50/50 beam splitters; loss channels are depicted in lighter font for better readability.

$Q$ but ensures that every eigenspace of $Q$ remains unaffected for all possible detection results. When $Q$ is degenerate, this only ensures that a state inside an eigenspace is sent to a state in the same eigenspace. A degeneracy-preserving quantum nondemolition (DP-QND) measurement is a QND measurement that does not affect any eigenstate of $Q$. In other words, the DP-QND measurement acts as the identity on each eigenspace [13]. Consider the parity observable $Q=Q_{+}-Q_{-}$for two qubits in the canonical basis $\{|0\rangle,|1\rangle\}$, with

$$
Q_{+}=|00\rangle\langle 00|+| 11\rangle\left\langle 11\left|, \quad Q_{-}=\right| 01\right\rangle\langle 01|+| 10\rangle\langle 10| .
$$

In a projective measurement of $Q$, detection result $+(-)$ would project the two qubits onto the even parity manifold $\operatorname{span}\{|00\rangle,|11\rangle\}$ (the odd parity manifold $\operatorname{span}\{|01\rangle,|10\rangle\}$ ). Less decisive DP-QND measurements can result, for instance, from classical detection uncertainty, e.g., with probability $1-\xi$ an even (odd) parity state gives detection result $-(+)$. Then the probability to detect \pm becomes $p_{ \pm}=\xi\left\langle\psi\left|Q_{ \pm}\right| \psi\right\rangle+$ $(1-\xi)\left\langle\psi\left|Q_{\mp}\right| \psi\right\rangle$ with corresponding measurement backaction transforming the initial state $|\psi\rangle$ into [29]

$$
\tilde{\mathbb{K}}_{ \pm}(|\psi\rangle\langle\psi|)=\frac{\xi Q_{ \pm}|\psi\rangle\left\langle\psi\left|Q_{ \pm}+(1-\xi) Q_{\mp}\right| \psi\right\rangle\langle\psi| Q_{\mp}}{p_{ \pm}} .
$$

Any even-parity (odd-parity) state remains unchanged under such measurement backaction and predominantly gives detection result $+(-)$, i.e., with probability $\xi \in(1 / 2,1]$.

We first sketch the general concept of our proposal for DP-QND measurement of parity, with $\left|\psi_{p}\right\rangle=\left|q_{p}\right\rangle$ an abstract probe qubit, similarly to the distillation discussion in e.g., Ref. [13]. Starting with $\left|q_{p}\right\rangle=|0\rangle_{p}$ we let $U_{A}\left(U_{B}\right)$ implement a CNOT gate on $\left|q_{p}\right\rangle$ conditioned by $\left|q_{A}\right\rangle\left(\left|q_{B}\right\rangle\right)$; see Fig. 1(b). If the channel $C$ was perfect $\left[E_{k}=\right.$ Identity for $k=1,2, \ldots$ in Fig. 1(b)], then an initial state $(|11\rangle \pm|00\rangle)_{A, B}|0\rangle_{p} / \sqrt{2}$ would remain unchanged, while an initial state $(|10\rangle \pm$ $|01\rangle)_{A, B}|0\rangle_{p} / \sqrt{2}$ would become $(|10\rangle \pm|01\rangle)_{A, B}|1\rangle_{p} / \sqrt{2}$ just before detection of the probe. Thus the measurement operations correspond to $\tilde{\mathbb{K}}_{+}, \tilde{\mathbb{K}}_{-}$with $\xi=1$.

Now let the channel subject the probe to an unknown number $n \in\{0,1,2, \ldots\}$ of bit flips $E_{k}=|1\rangle\left\langle\left. 0\right|_{p}+\mid 0\right\rangle\left\langle\left. 1\right|_{p}\right.$; this can be represented as conditioning each bit flip on an unknown hypothetical state of the environment. For $n$ even, the outcome is as for the perfect channel. For $n$ odd the final probe state is reversed, e.g., input $\left(|11\rangle \pm|00\rangle_{A, B}|0\rangle_{p} / \sqrt{2}\right.$ yields output $(|11\rangle \pm|00\rangle)_{A, B}|1\rangle_{p} / \sqrt{2}$, but most importantly, the target qubits' state remains unaffected. This essential property ensures that the expected evolution for $n$ unknown (equivalently, tracing over the unknown states of the environment) remains a DP-QND parity measurement, explicitly described by operators $\tilde{\mathbb{K}}_{+}, \tilde{\mathbb{K}}_{-}$with $\xi=\sum_{n \text { even }} \operatorname{Prob}(n)<1$. One can easily adapt this $\xi$ to include detection misses and errors. A broad distribution of values of $n$ implies low measurement contrast, pushing $\xi$ close to $1 / 2$, but it does not impede its DP-QND character. Hence when sufficiently many measurements can be repeated within a relevant timescale, a conclusive result is obtained even for $\xi$ close to $1 / 2$ (see details below).

This DP-QND property is, in fact unavoidably, lost under general channel errors (see Appendix A). If, for instance, $C$ includes a phase flip $E_{1}=|0\rangle\langle 0|-| 1\rangle\left\langle\left. 1\right|_{p}\right.$, then the initial even-parity Bell state $\left|\psi_{+}\right\rangle=(|11\rangle+|00\rangle)_{A, B} / \sqrt{2}$ gets transformed by measurement backaction into $\left|\psi_{-}\right\rangle=(|11\rangle-$ $|00\rangle)_{A, B} / \sqrt{2}$; thus this eigenstate of $Q$ is not conserved, breaking the DP-QND character. Not knowing if $E_{1}$ was applied or not, the initial maximally entangled state $\left|\psi_{+}\right\rangle$gets transformed into a statistical mixture of $\left|\psi_{+}\right\rangle$and $\left|\psi_{-}\right\rangle$, i.e., entanglement is lost. 


\section{TOWARDS A PHYSICAL IMPLEMENTATION}

These abstract properties indicate a pathway towards losstolerant parity measurement: use a subspace of probe states on which the dominant decoherence channel acts as a unitary root of identity, e.g., a bit flip. Our key observation is that this fits a physical implementation where the probe is an electromagnetic field pulse whose logical states are materialized by so-called "cat states", i.e., mesoscopic superpositions of coherent states. This encoding, proposed earlier for quantum computing $[26,30]$, ensures that photon losses imply to a good approximation coherent logical bit flips. The rest of this paper describes such implementation in detail.

\section{A. Probe's cat states}

This physical implementation of loss-tolerant parity measurement is sketched in Fig. 1(c). We denote by

$$
\left|\mathcal{C}_{\beta}^{ \pm}\right\rangle=(|\beta\rangle \pm|-\beta\rangle) / \mathcal{N}_{\beta}^{ \pm}, \quad \mathcal{N}_{\beta}^{ \pm}=\sqrt{2 \pm 2 e^{-2|\beta|^{2}}},
$$

the superpositions between two coherent states $|\beta\rangle$ and $|-\beta\rangle, \beta \in \mathbb{R}$; the normalization constants $\mathcal{N}_{\beta}^{ \pm}$rapidly approach $\sqrt{2}$ as the coherent amplitude $\beta$ increases. The probe field is initially prepared in $\left|\mathcal{C}_{\alpha}^{+}\right\rangle_{p}$ and interacts with two qubitcavity systems in a cascaded manner. Between the two setups it is exposed to losses, modeled as mixing with the vacuum state $|0\rangle_{\mathrm{env}}$ of an ancillary mode through a beam-splitter unitary operator $U_{\mathrm{BS}}^{\eta}$ with transmittance $\sqrt{\eta}$ and reflectance $\sqrt{1-\eta}$. The unitary operators apply:

$$
\begin{aligned}
U_{A}|0\rangle_{A}\left|\mathcal{C}_{\alpha}^{ \pm}\right\rangle_{p} & =|0\rangle_{A}\left|\mathcal{C}_{\alpha}^{ \pm}\right\rangle_{p}, \\
U_{A}|1\rangle_{A}\left|\mathcal{C}_{\alpha}^{ \pm}\right\rangle_{p} & =|1\rangle_{A}\left|\mathcal{C}_{\alpha}^{\mp}\right\rangle_{p}, \\
U_{B}|0\rangle_{B}\left|\mathcal{C}_{\sqrt{\eta} \alpha}^{ \pm}\right\rangle_{p} & =|0\rangle_{B}\left|\mathcal{C}_{\sqrt{\eta} \alpha}^{ \pm}\right\rangle_{p}, \\
U_{B}|1\rangle_{B}\left|\mathcal{C}_{\sqrt{\eta} \alpha}^{ \pm}\right\rangle_{p} & =|1\rangle_{B}\left|\mathcal{C}_{\sqrt{\eta} \alpha}^{\mp}\right\rangle_{p}, \\
U_{\mathrm{BS}}^{\eta}\left|\mathcal{C}_{\alpha}^{ \pm}\right\rangle_{p}|0\rangle_{\mathrm{env}} & =\frac{1}{\mathcal{N}_{\alpha}^{ \pm}}\left(|\sqrt{\eta} \alpha\rangle_{p}|\sqrt{1-\eta} \alpha\rangle_{\mathrm{env}}\right. \\
& \left. \pm|-\sqrt{\eta} \alpha\rangle_{p}|-\sqrt{1-\eta} \alpha\rangle_{\mathrm{env}}\right) .
\end{aligned}
$$

Finally, after interaction with the second qubit, a measurement (e.g., of photon-number parity observable) projects the probe's state onto $\left|\mathcal{C}_{\sqrt{\eta} \alpha}^{+}\right\rangle_{p}$ or $\left|\mathcal{C}_{\sqrt{\eta} \alpha}^{-}\right\rangle_{p}$. Calling $\left|\mathcal{C}_{\beta}^{+}\right\rangle_{p}$ and $\left|\mathcal{C}_{\beta}^{-}\right\rangle_{p}$ respectively the logical $|0\rangle_{p}$ and $|1\rangle_{p}$, we recover the scheme where $U_{A}, U_{B}$ implement CNOT gates.

Before analyzing in detail the cascade scheme of Fig. 1(c), we want to introduce a corresponding concurrent scheme, depicted in Fig. 1(d). The initial 50/50 beam splitter generates a probe state of two entangled fields, $\left(\left|\mathcal{C}_{\alpha}^{+}\right\rangle_{p A}\left|\mathcal{C}_{\alpha}^{-}\right\rangle_{p B}+\right.$ $\left.\left|\mathcal{C}_{\alpha}^{-}\right\rangle_{p A}\left|\mathcal{C}_{\alpha}^{+}\right\rangle_{p B}\right) / \sqrt{2}$, with $\alpha=\gamma / \sqrt{2}$. These two output fields are sent along lossy quantum channels towards the respective qubits where they undergo the equivalent of a CNOT. After the final beam-splitter, a similar measurement to the cascaded scheme is applied to the channel $A^{\prime}$. While the rest of the paper will deal with the analysis of the cascaded scheme, all the derivations can be adapted to this concurrent architecture in a straightforward manner.

\section{B. Performance analysis}

We now analyze in detail the performance of the scheme of Fig. 1(c). As we trace on the ancillary field modeling the losses, we can without loss of generality imagine a virtual detector also projecting it to $\left|\mathcal{C}_{\sqrt{1-\eta} \alpha}^{+}\right\rangle_{\text {env }}$ or $\left|\mathcal{C}_{\sqrt{1-\eta} \alpha}^{-}\right\rangle_{\text {env }}$, but with unread detection result. The measurement outcomes of the two detectors (one real and one virtual) are associated with four Kraus operators $M_{\sigma, \sigma}$ and $M_{\sigma,-\sigma}$ with $\sigma \in\{+,-\}$, modeling the backaction of the measurements on the target qubits: e.g., their state $\rho$, after measuring even parities for both detectors, is modified to $M_{+,+} \rho M_{+,+}^{\dagger} / \operatorname{Tr}\left(M_{+,+} \rho M_{+,+}^{\dagger}\right)$. Following the computations of Appendix B, these Kraus operators are

$$
\begin{aligned}
M_{\sigma, \sigma} & =\frac{\mathcal{N}_{\sqrt{1-\eta} \alpha}^{\sigma}}{2}\left(\frac{\mathcal{N}_{\sqrt{\eta} \alpha}^{\sigma}}{\mathcal{N}_{\alpha}^{+}}|00\rangle\left\langle 00\left|+\frac{\mathcal{N}_{\sqrt{\eta} \alpha}^{(-\sigma)}}{\mathcal{N}_{\alpha}^{-}}\right| 11\right\rangle\langle 11|\right), \\
M_{\sigma,-\sigma} & =\frac{\mathcal{N}_{\sqrt{1-\eta} \alpha}^{(-\sigma)}}{2}\left(\frac{\mathcal{N}_{\sqrt{\eta} \alpha}^{\sigma}}{\mathcal{N}_{\alpha}^{-}}|10\rangle\left\langle 10\left|+\frac{\mathcal{N}_{\sqrt{\eta} \alpha}^{(-\sigma)}}{\mathcal{N}_{\alpha}^{+}}\right| 01\right\rangle\langle 01|\right) .
\end{aligned}
$$

Discarding the inaccessible outcome of the virtual detector, the backaction induced by the probe field measurement follows:

$$
\begin{aligned}
\mathbb{K}_{+}(\rho) & =\frac{M_{+,+} \rho M_{+,+}^{\dagger}+M_{+,-} \rho M_{+,-}^{\dagger}}{\operatorname{Tr}\left(M_{+,+} \rho M_{+,+}^{\dagger}+M_{+,-} \rho M_{+,-}^{\dagger}\right)}, \\
\mathbb{K}_{-}(\rho) & =\frac{M_{-,+} \rho M_{-,+}^{\dagger}+M_{-,-} \rho M_{-,-}^{\dagger}}{\operatorname{Tr}\left(M_{-,+} \rho M_{-,+}^{\dagger}+M_{-,-} \rho M_{-,-}^{\dagger}\right)} .
\end{aligned}
$$

In the lossless case $(\eta=1), M_{+,-}$and $M_{-,-}$vanish as $\mathcal{N}_{0}^{-}=0$; the coefficients in front of $|00\rangle\langle 00|$ and $|11\rangle\langle 11|$ in $M_{+,+}$and in front of $|01\rangle\langle 01|$ and $|10\rangle\langle 10|$ in $M_{-,+}$are identical, equal to 1 . This is a projective parity measurement, as described by $\widetilde{\mathbb{K}}_{+}, \widetilde{\mathbb{K}}_{-}$with $\xi=1$.

The effect of transmission losses $(\eta<1)$ is twofold. First, it reduces the measurement strength. Indeed, when the probe field is detected in a given parity (e.g., +), the qubits could be projected to the opposite parity (e.g., by the Kraus operator $\left.M_{+,-}\right)$. However, each measurement does increase the conditional probability of finding the qubits in the same parity as the one indicated by the detections, because

$$
\begin{aligned}
& \mathcal{N}_{\sqrt{1-\eta} \alpha}^{+} \min \left(\frac{\mathcal{N}_{\sqrt{\eta} \alpha}^{+}}{\mathcal{N}_{\alpha}^{+}}, \frac{\mathcal{N}_{\sqrt{\eta} \alpha}^{-}}{\mathcal{N}_{\alpha}^{-}}\right) \\
& >\mathcal{N}_{\sqrt{1-\eta} \alpha}^{-} \max \left(\frac{\mathcal{N}_{\sqrt{\eta} \alpha}^{+}}{\mathcal{N}_{\alpha}^{-}}, \frac{\mathcal{N}_{\sqrt{\eta} \alpha}^{-}}{\mathcal{N}_{\alpha}^{+}}\right) .
\end{aligned}
$$

A projective parity measurement under perfect transmission $(\eta=1)$ is thus replaced by a less decisive measurement for $\eta<1$, where at each shot we gain partial information on the parity. By repeating the measurement the state gets projected onto a well-defined parity subspace. Most importantly, we preserve the QND property: an initial state of definite parity always keeps this parity [e.g., $M_{+,-}(|00\rangle+|11\rangle)=0$ ].

However, the second, more harmful effect of transmission loss is a slight perturbation of the DP-QND property, by introducing slow mixing within each parity subspace. This is due to the coherent states $|\beta\rangle$ and $|-\beta\rangle$ not being perfectly 


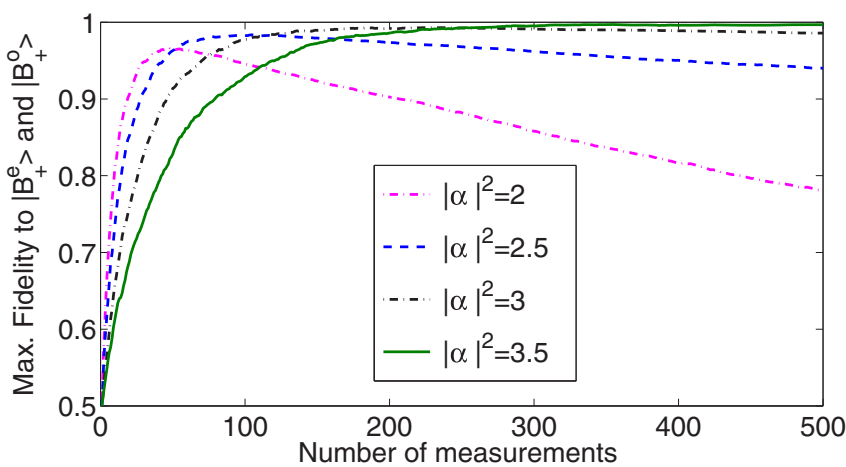

FIG. 2. Average evolution, over 1000 Monte Carlo simulations, of the initial state $(|0\rangle+|1\rangle)_{A}(|0\rangle+|1\rangle)_{B} / 2$ under repeated approximate DP-QND parity measurements (2), (3), for various probe field intensities $|\alpha|^{2}$ and transmission efficiency $\eta=0.75$. The state initially converges towards a definite parity state $\left|B_{+}^{e}\right\rangle=(|00\rangle+$ $|11\rangle) / \sqrt{2}$ or $\left|B_{+}^{o}\right\rangle=(|01\rangle+|10\rangle) / \sqrt{2}$ thanks to the measurement, then slowly loses fidelity to those states by dephasing, due to the slight perturbation of the DP-QND property inside definite parity subspaces. Here and everywhere throughout the paper, the fidelity to a Bell state $\left|B_{+}^{e}\right\rangle$ is defined as $\left\langle B_{+}^{e}|\rho| B_{+}^{e}\right\rangle$.

orthogonal, so that $\mathcal{N}_{\beta}^{+} \neq \mathcal{N}_{\beta}^{-}$. This effectively induces dephasing inside parity subspaces, e.g., $\mathcal{N}_{\sqrt{\eta} \alpha}^{+} / \mathcal{N}_{\alpha}^{+}>\mathcal{N}_{\sqrt{\eta} \alpha}^{-} / \mathcal{N}_{\alpha}^{-}$ implies that $M_{+,+}$drives $\left|B_{ \pm}^{e}\right\rangle=(|00\rangle \pm|11\rangle) / \sqrt{2}$ closer to $|00\rangle=\left(\left|B_{+}^{e}\right\rangle+\left|B_{-}^{e}\right\rangle\right) / \sqrt{2}$, while $M_{-,-}$would drive them closer to $|11\rangle=\left(\left|B_{+}^{e}\right\rangle-\left|B_{-}^{e}\right\rangle\right) / \sqrt{2}$.

The simulations in Fig. 2 illustrate the competition between parity measurement and undesired dephasing for various $|\alpha|^{2}$ (average number of photons in the probe field). Initializing both qubits in the state $\left|+_{X}\right\rangle=(|0\rangle+|1\rangle) / \sqrt{2}$, a DP-QND parity measurement should project the joint state towards one of the two Bell states $\left|B_{+}^{e}\right\rangle=(|00\rangle+|11\rangle) / \sqrt{2}$ or $\left|B_{+}^{o}\right\rangle=$ $(|01\rangle+|10\rangle) / \sqrt{2}$. This is the dominant tendency in Fig. 2, but transmission loss induces slow dephasing, mixing the target Bell states with the undesired ones $\left|B_{-}^{e}\right\rangle=(|00\rangle-$ $|11\rangle) / \sqrt{2}$ and $\left|B_{-}^{o}\right\rangle=(|01\rangle-|10\rangle) / \sqrt{2}$. By increasing $|\alpha|^{2}$, the difference between $\mathcal{N}_{\sqrt{\eta} \alpha}^{+} / \mathcal{N}_{\alpha}^{+}$and $\mathcal{N}_{\sqrt{\eta} \alpha}^{-} / \mathcal{N}_{\alpha}^{-}$decreases and this undesired dephasing gets suppressed significantly, at the expense of slower convergence, i.e., weaker parity measurement. As soon as $\eta>1 / 2$, one can achieve arbitrarily high fidelity in this way.

In the next section, we give approximate analytical formulas for these two competing rates, providing the maximal achievable fidelity as a function of $\eta,|\alpha|^{2}$ and qubit lifetimes.

\section{Measurement and dephasing rates}

The rate at which the parity measurement acquires information can be expressed analytically as a function of $\eta$ and $|\alpha|^{2}$. Define the Lyapunov function

$$
\begin{aligned}
V^{\text {parity }}(\rho)= & \sqrt{\langle 00|\rho| 00\rangle\langle 10|\rho| 10\rangle} \\
& +\sqrt{\langle 11|\rho| 11\rangle\langle 01|\rho| 01\rangle} \\
= & \frac{w_{B 0}}{2} \sqrt{1-\left(P_{B 0}\right)^{2}}+\frac{w_{B 1}}{2} \sqrt{1-\left(P_{B 1}\right)^{2}},
\end{aligned}
$$

where $w_{B q_{B}}=\left\langle q_{B}|\rho| q_{B}\right\rangle_{B}$ denotes the population with qubit $B$ in state $\left|q_{B}\right\rangle$ and $P_{B q_{B}}=\operatorname{Tr}\left(\sigma_{z} \otimes \sigma_{z} \rho\left|q_{B}\right\rangle\left\langle q_{B}\right|\right) / w_{B q_{B}}$ measures the parity, conditioned on qubit $B$ being in state $\left|q_{B}\right\rangle$, for $q_{B} \in\{0,1\}$. One computes that

$$
\left\langle V^{\text {parity }}\left(\rho_{k+1}\right)\right\rangle=\frac{\sqrt{1-e^{-4(1-\eta)|\alpha|^{2}}}}{\sqrt{1-e^{-4|\alpha|^{2}}}}\left\langle V^{\text {parity }}\left(\rho_{k}\right)\right\rangle,
$$

where $\rho_{k}$ is the joint qubits state after the $k$ th measurement and $\left\langle V^{\text {parity }}\right\rangle$ denotes the ensemble average of $V^{\text {parity }}$ over measurement realizations. Indeed, we have

$$
\begin{aligned}
\mathbb{E}\left(V^{\text {parity }}\left(\rho_{k+1}\right) \mid \rho_{k}\right)= & V^{\text {parity }}\left(\mathbb{K}_{+}\left(\rho_{k}\right)\right) \mathbb{P}\left(+\mid \rho_{k}\right) \\
& +V^{\text {parity }}\left(\mathbb{K}_{-}\left(\rho_{k}\right)\right) \mathbb{P}\left(-\mid \rho_{k}\right),
\end{aligned}
$$

where

$$
\begin{aligned}
& \mathbb{P}\left(+\mid \rho_{k}\right)=\operatorname{Tr}\left(M_{+,+} \rho_{k} M_{+,+}^{\dagger}+M_{+,-} \rho_{k} M_{+,-}^{\dagger}\right), \\
& \mathbb{P}\left(-\mid \rho_{k}\right)=\operatorname{Tr}\left(M_{-,+} \rho_{k} M_{-,+}^{\dagger}+M_{-,-} \rho_{k} M_{-,-}^{\dagger}\right)
\end{aligned}
$$

are respectively the conditional probabilities of achieving a positive or negative outcome at $k$ th measurement. From this, we get

$$
\begin{aligned}
\mathbb{E}( & \left.V^{\text {parity }}\left(\rho_{k+1}\right) \mid \rho_{k}\right) \\
= & \sqrt{\left\langle 00\left|M_{+,+} \rho_{k} M_{+,+}^{\dagger}\right| 00\right\rangle\left\langle 10\left|M_{+,-} \rho_{k} M_{+,-}^{\dagger}\right| 10\right\rangle} \\
& +\sqrt{\left\langle 11\left|M_{+,+} \rho_{k} M_{+,+}^{\dagger}\right| 11\right\rangle\left\langle 01\left|M_{+,-} \rho_{k} M_{+,-}^{\dagger}\right| 01\right\rangle} \\
& +\sqrt{\left\langle 00\left|M_{-,-} \rho_{k} M_{-,-}^{\dagger}\right| 00\right\rangle\left\langle 10\left|M_{-,+} \rho_{k} M_{-,+}^{\dagger}\right| 10\right\rangle} \\
& +\sqrt{\left\langle 11\left|M_{-,-} \rho_{k} M_{-,-}^{\dagger}\right| 11\right\rangle\left\langle 01\left|M_{-,+} \rho_{k} M_{-,+}^{\dagger}\right| 01\right\rangle} \\
= & \left(\frac{\mathcal{N}_{\sqrt{1-\eta} \alpha}^{+} \mathcal{N}_{\sqrt{1-\eta} \alpha}^{-}}{\mathcal{N}_{\alpha}^{+} \mathcal{N}_{\alpha}^{-}}\right) V^{\text {parity }}\left(\rho_{k}\right) .
\end{aligned}
$$

Taking the expectation value of both sides with respect to $\rho_{k}$, we find the result of Eq. (5). Thus, $V^{\text {parity }}$ exponentially decays to zero at a rate (per measurement iteration)

$$
r_{\text {parity }}=\frac{1}{2} \ln \left(\frac{1-e^{-4|\alpha|^{2}}}{1-e^{-4(1-\eta)|\alpha|^{2}}}\right) .
$$

In the case of a fully DP-QND measurement, i.e., assuming $\mathcal{N}_{\sqrt{\eta} \alpha}^{+} / \mathcal{N}_{\alpha}^{+} \simeq \mathcal{N}_{\sqrt{\eta} \alpha}^{-} / \mathcal{N}_{\alpha}^{-}$and $\mathcal{N}_{\sqrt{\eta} \alpha}^{+} / \mathcal{N}_{\alpha}^{-} \simeq \mathcal{N}_{\sqrt{\eta} \alpha}^{-} / \mathcal{N}_{\alpha}^{+}$in the Kraus operators, the two terms in the Lyapunov function (4) would decay at the same rate and $r_{\text {parity }}$ represents precisely the parity measurement strength. Indeed, in this case we would obtain the same result with the alternative Lyapunov function $V_{\text {ideal }}^{\text {parity }}(\rho)=\frac{1}{2}\left[1-P(\rho)^{2}\right]^{1 / 2}$, where $P(\rho)=\operatorname{Tr}\left(\sigma_{z} \otimes \sigma_{z} \rho\right)$.

One can also analytically calculate the dephasing rate induced by the transmission loss. To this aim we define the coherence function

$$
C(\rho)=|\langle 00|\rho| 11\rangle|+|\langle 01|\rho| 10\rangle| .
$$

Similar calculations as above lead to

$$
\left\langle C\left(\rho_{k+1}\right)\right\rangle=\frac{\sqrt{1-e^{-4 \eta|\alpha|^{2}}}}{\sqrt{1-e^{-4|\alpha|^{2}}}}\left\langle C\left(\rho_{k}\right)\right\rangle .
$$




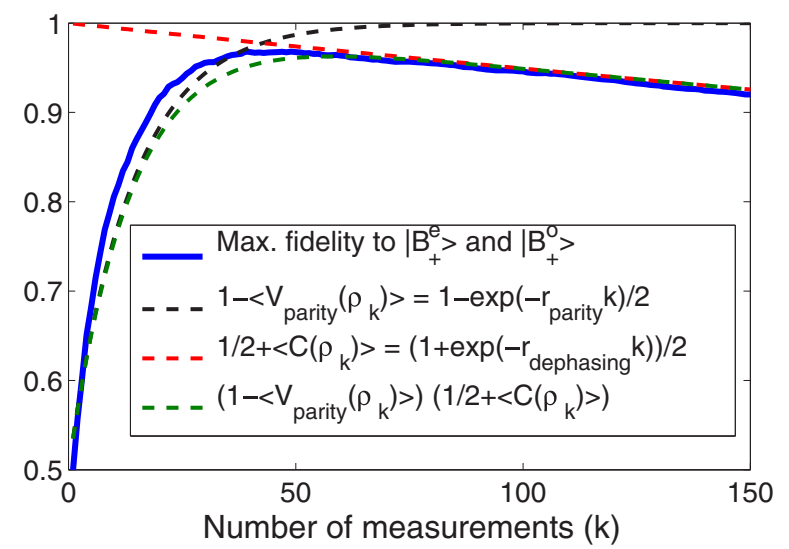

FIG. 3. The performance of the near DP-QND parity measurement is explained by two rates. The measurement strength $r_{\text {parity }}$ indicates how fast the state is projected onto a parity eigenspace. The dephasing rate $r_{\text {dephasing }}$ indicates how fast the coherence inside a parity eigenspace vanishes. Here, we have fixed $\eta=0.75$ and $|\alpha|^{2}=2$. The blue (solid) curve illustrates the maximum fidelity to one of the two Bell states $\left|B_{+}^{e}\right\rangle$ and $\left|B_{+}^{o}\right\rangle$, in simulations of our measurement scheme, when the two qubits are initialized in the state $(|0\rangle+|1\rangle)_{A}(|0\rangle+$ $|1\rangle)_{B} / 2$ and we take an average over 1000 Monte Carlo trajectories. The black (dashed) curve illustrates the average parity converging at the characteristic rate of the Lyapunov function $V_{\text {parity }}(\rho)$. The red (dashed) curve, decreasing with the same characteristic rate as the coherence function $C(\rho)$, illustrates the dephasing inside a parity eigenspace. The green (dashed) curve represents the product of both these effects. This theoretical curve fits reasonably well with the simulations (blue solid curve).

Therefore the coherence function $C(\rho)$ exponentially decays to zero at a rate (per measurement iteration)

$$
r_{\text {dephasing }}=\frac{1}{2} \ln \left(\frac{1-e^{-4|\alpha|^{2}}}{1-e^{-4 \eta|\alpha|^{2}}}\right) .
$$

Figure 3 provides a comparison of the simulations to the above analytical results. As can be seen, the performance of the parity measurement protocol can be well explained by using the above two rates. The slight mismatch between the raising rate of the blue curve (fidelity to Bell states) and the theoretical $r_{\text {parity }}$ can be explained by the nonlinear relation between the fidelity and the Lyapunov function $V_{\text {parity. This }}$ nonlinearity makes it impossible to translate the rate $r_{\text {parity }}$ into a precise exponential rate for the raising dynamics of the average fidelity.

The expressions for $r_{\text {parity }}$ and $r_{\text {dephasing }}$ allow us to analyze the measurement performance as a function of $\eta$ and $|\alpha|^{2}$. Consider again the evolution depicted in Figs. 2 and 3. The fidelity to the closest Bell state is $1 / 2$ times the sum of two terms: dominant parity population, which converges from $1 / 2$ to 1 at roughly a rate $r_{\text {parity }}$, and dominant phase population, which decreases from 1 to $1 / 2$ at a rate $r_{\text {dephasing }}$. The two terms contribute equally to the error after a number of measurements $\tau=\tau_{\text {meas }}$ satisfying $e^{-r_{\text {parity }} \tau}+e^{-r_{\text {dephasing }} \tau}=1$. The corresponding estimate of Bell-state fidelity is $F_{\text {meas }}=$ $1-e^{-r_{\text {parity }} \tau_{\text {meas }}} / 2$. This is a good estimate of the maximum reached by the curves on Figs. 2 and 3. Figure 4 illustrates these indicators of our parity measurement's performance. For

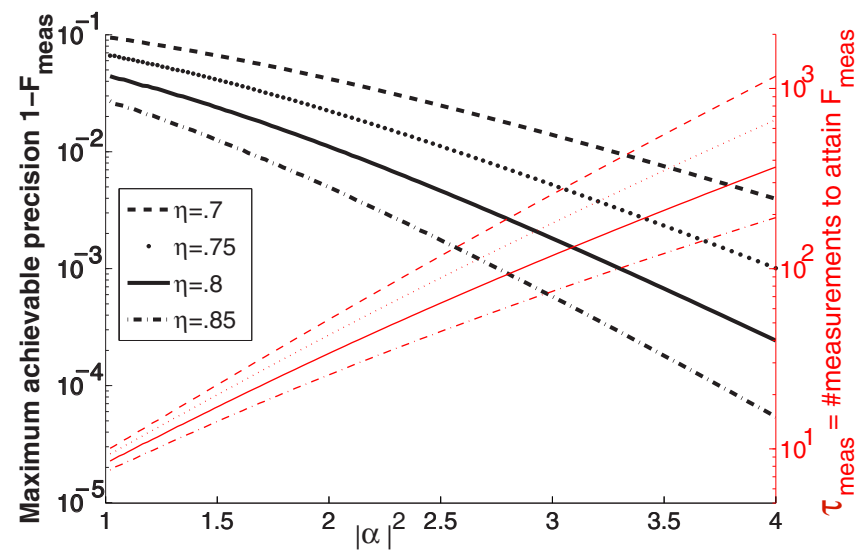

FIG. 4. Estimate $F_{\text {meas }}$ of the highest fidelity to the closest Bell state (thick black, left axis), obtained by repeated application of our near DP-QND parity measurement (2), (3) starting from $(|0\rangle+|1\rangle)_{A}(|0\rangle+|1\rangle)_{B} / 2$, before dephasing destroys coherence in absence of feedback. Estimate $\tau_{\text {meas }}$ of the number of measurements after which this highest fidelity is reached (thin red, right axis), giving an indication of measurement strength.

transmission efficiency as low as $70 \%$ we can get $F_{\text {meas }}$ as high as $99 \%$ in 400 measurement steps, taking $|\alpha|^{2}=3.273$. Increasing $\eta$ to $85 \%$ one achieves the same $F_{\text {meas }}$ with only 17 measurements, taking $|\alpha|^{2}=1.63$.

In the limit $r_{\text {parity }} \gg r_{\text {dephasing }}$ (equivalent to $e^{4(2 \eta-1)|\alpha|^{2}} \gg 1$ ), one can approximately replace $e^{-r_{\text {dephasing }} \tau}$ by $1-r_{\text {dephasing }} \tau$, and therefore the solution to the transcendental equation $e^{-r_{\text {parity }} \tau}+$ $e^{-r_{\text {dephasing }} \tau}=1$, giving the point of maximal fidelity, is well estimated by

$$
\tau_{\text {meas }}=\frac{1}{r_{\text {parity }}} W_{0}\left(\frac{r_{\text {parity }}}{r_{\text {dephasing }}}\right),
$$

where $W_{0}$ is the Lambert $W$ function.

\section{FEEDBACK STABILIZATION OF BELL STATES}

Our near DP-QND parity measurement can be used to stabilize a particular Bell state with a simple feedback protocol. To stabilize $\left|B_{+}^{e}\right\rangle$, (i) apply a $\pi$ pulse around the $X$ axis on the first qubit whenever the measurements estimate a probability higher than $1 / 2$ to be in the odd-parity subspace, (ii) after that, apply a $\pi / 2$ pulse on both qubits around the $Y$ axis irrespectively of the detection result. The measurement backaction favors convergence towards the dominant parity, the $\pi$ pulse correcting the parity whenever this is not even. This pushes the state towards the span of $\left|B_{+}^{e}\right\rangle,\left|B_{-}^{e}\right\rangle$ without favoring the target $\left|B_{+}^{e}\right\rangle$. The two $\pi / 2$ pulses then leave $\left|B_{+}^{e}\right\rangle$ untouched and send the undesired $\left|B_{-}^{e}\right\rangle$ onto $\left|B_{+}^{o}\right\rangle$, such that the next parity measurement stochastically moves the corresponding population as well towards the target. Simulations in Fig. 5 illustrate the performance of this protocol, having fixed $\eta=0.75$ and varying $|\alpha|^{2}$. As expected, a larger $|\alpha|^{2}$ allows us to reach higher final fidelity but also implies slower convergence.

The information needed to implement general feedback decisions is typically obtained by a quantum filter, i.e., a 


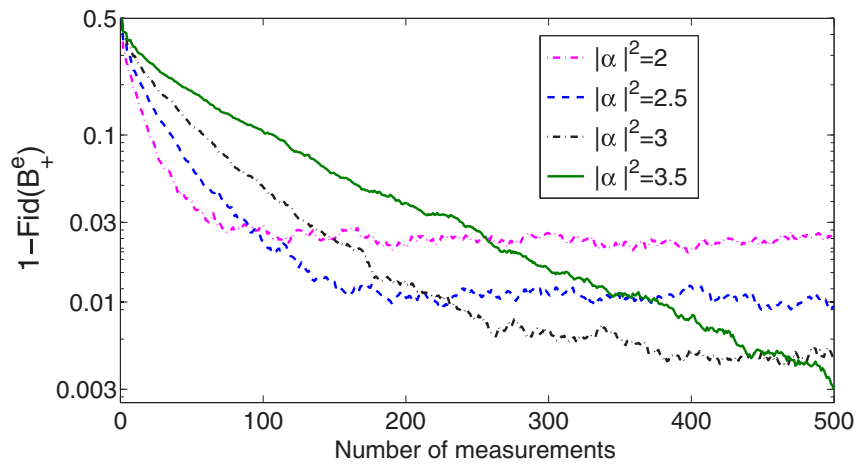

FIG. 5. Feedback stabilization of Bell state $\left|B_{+}^{e}\right\rangle$.

computer estimating the state by simulating the evolution (3) associated with the respective detection results. Such filters are known to be stable [31]. In the present case, because the feedback decision requires only to know the respective parity populations, the quantum filter can be simplified significantly; namely, by discarding all off-diagonal components in the Bell-state basis. Indeed, first note that the measurement does not depend on coherences among subspaces of different parity. Moreover, the measurement itself completely destroys, in a single iteration, any coherences between subspaces of different parity. The $\pi / 2$ pulses "export" coherences possibly present between, e.g., $\left|B_{+}^{e}\right\rangle$ and $\left|B_{-}^{e}\right\rangle$ into coherences between $\left|B_{+}^{e}\right\rangle$ and $\left|B_{+}^{o}\right\rangle$, i.e., between different parity subspaces, which are thus destroyed at the next measurement; while they "import" into, e.g., the even-parity eigenspace, the coherences previously present between $\left|B_{+}^{e}\right\rangle$ and $\left|B_{+}^{o}\right\rangle$, which are none since those two Bell states belong to different-parity eigenspaces and their coherences were thus destroyed by the last measurement. Thus, after one initial weak measurement step at most, no relevant coherences among Bell states will survive. This allows us to update just the populations on the four Bell states, as in a classical filter for a partially observed Markov chain. This filter, updating the populations on the four Bell states, is the only computation required for the feedback: the action itself just requires a binary decision, to switch towards the predominantly populated parity.

To analyze the feedback more explicitly, we note that instead of applying the $\pi / 2$ pulses to rotate the state in the Schrödinger picture, we can reformulate the dynamics by applying the $\pi / 2$ pulses to the measurement scheme, in an equivalent Heisenberg picture. The corresponding measurements then alternate between the parity measurement $\sigma_{z} \otimes \sigma_{z}$ in the $z$ basis, and a parity measurement $\sigma_{x} \otimes \sigma_{x}$ in the $x$ basis. The corresponding state is subject to $\pi$ pulses in the respective $z$ or $x$ basis. A $\pi$ pulse in the $z$ basis ( $x$ basis) does not change the populations of $x$-parity subspaces (of $z$-parity subspaces) and is applied to increase the population in $\operatorname{span}\left\{\left|B_{+}^{e}\right\rangle,\left|B_{-}^{e}\right\rangle\right\}\left(\operatorname{span}\left\{\left|B_{+}^{e}\right\rangle,\left|B_{+}^{o}\right\rangle\right\}\right)$. The stochastic convergence of the measured system towards a definite parity in both $x$ and $z$ coordinates ensures that, in the absence of other effects, the population in $\operatorname{span}\left\{\left|B_{+}^{e}\right\rangle,\left|B_{-}^{e}\right\rangle\right\} \cap$ $\operatorname{span}\left\{\left|B_{+}^{e}\right\rangle,\left|B_{+}^{o}\right\rangle\right\}=\left|B_{+}^{e}\right\rangle$ would increase in expectation until reaching $100 \%$. The dephasing effect limits the actual fidelity for a given $\alpha$. Taking $\alpha$ larger allows us to increase the fidelity, but slows down the convergence, such that a trade-off value of $\alpha$ must be selected with respect to other decoherence effects acting on the system.

We can quantify the performance of this feedback scheme by using the characteristic convergence rates computed in the previous section. Noting that the $\pi / 2$ pulses ensure a rapid oscillation between the states $\left|B_{-}^{e}\right\rangle$ and $\left|B_{+}^{o}\right\rangle$, we define $\xi(t)$ to be their combined population at time $t$. For $r_{\text {parity }}, r_{\text {dephasing }} \ll 1$ i.e., a small rate of change per measurement iteration, we can approximate the system dynamics by a continuous-time model for the populations $p_{k}$ of Bell state $|k\rangle\left(\right.$ and $\left.\xi=p_{B_{-}^{e}}+p_{B_{+}^{o}}\right)$ :

$$
\begin{aligned}
\frac{d}{d t} p_{B_{+}^{e}}= & -\frac{r_{\text {dephasing }}}{2 T_{d}} p_{B_{+}^{e}}+\left(\frac{r_{\text {parity }}}{2 T_{d}}+\frac{r_{\text {dephasing }}}{4 T_{d}}\right) \xi, \\
\frac{d}{d t} p_{B_{-}^{o}}= & -\left(\frac{r_{\text {parity }}}{T_{d}}+\frac{r_{\text {dephasing }}}{2 T_{d}}\right) p_{B_{-}^{o}}+\frac{r_{\text {dephasing }}}{4 T_{d}} \xi, \\
\frac{d}{d t} \xi= & -\left(\frac{r_{\text {parity }}}{2 T_{d}}+\frac{r_{\text {dephasing }}}{2 T_{d}}\right) \xi+\frac{r_{\text {dephasing }}}{2 T_{d}} p_{B_{+}^{e}} \\
& +\left(\frac{r_{\text {parity }}}{T_{d}}+\frac{r_{\text {dephasing }}}{2 T_{d}}\right) p_{B_{-}^{o}} .
\end{aligned}
$$

Here $t$ denotes actual time and $T_{d}$ is the time between two consecutive detection results. To obtain these equations, we have used the fact that the measurement destroys any coherences among Bell states, so only populations are relevant. The nonlinear relation between $V^{\text {parity }}$ and the fidelities makes it impossible to be more accurate than order of magnitude about $r_{\text {parity }}$, and we have observed in simulations that the true dynamics is more complicated than the linear model (6). However, the model (6) gives valuable indications about the dependence of the whole scheme on $\alpha$ (through $r_{\text {parity }}$ and $r_{\text {dephasing }}$ ) and allows us to efficiently optimize $\alpha$ for given settings. The steady state of Eq. (6) is obtained with

$$
p_{B_{+}^{e}}=\frac{\delta^{2}}{(1+\delta)^{2}}, \quad \text { where } \delta=\frac{2 r_{\text {parity }}}{r_{\text {dephasing }}}+1
$$

which increases with $\alpha$ as expected.

To practically illustrate the trade-off with slower convergence at larger $\alpha$, we can add to the picture the qubit relaxation and qubit dephasing:

$$
\begin{aligned}
\frac{d}{d t} \rho= & \sum_{k \in\{A, B\}} L_{k} \rho L_{k}^{\dagger}-\frac{1}{2}\left(L_{k}^{\dagger} L_{k} \rho+\rho L_{k}^{\dagger} L_{k}\right) \\
& +\sum_{k \in\{A, B\}} \tilde{L}_{k} \rho \tilde{L}_{k}^{\dagger}-\frac{1}{2}\left(\tilde{L}_{k}^{\dagger} \tilde{L}_{k} \rho+\rho \tilde{L}_{k}^{\dagger} \tilde{L}_{k}\right), \\
\text { with } \quad L_{k}= & \sqrt{\frac{1}{T_{1}^{k}}}|0\rangle\left\langle\left. 1\right|_{k}, \quad \tilde{L}_{k}=\sqrt{\frac{1}{2 T_{\phi}^{k}}} \sigma_{z}^{k} .\right.
\end{aligned}
$$

Here $|0\rangle\left\langle\left. 1\right|_{k}\right.$ is the qubit $k$ 's lowering operator, $\sigma_{z}^{k}$ is just $\sigma_{z}$ on qubit $k, T_{1}^{k}$ is the relaxation time, and $T_{\phi}^{k}$ is the pure dephasing time. Note that we apply these decoherences in the laboratory coordinates, where the qubits undergo $\pi / 2$ rotations after each measurement. One then computes that, in the Bell state basis, when discarding off-diagonal terms in $\rho$, the effect of this qubit 
decoherence is equivalent to replacing Eq. (6) by

$$
\begin{aligned}
\frac{d}{d t} p_{B_{+}^{e}} & =-\frac{\Gamma_{1}+\Gamma_{2}}{2} p_{B_{+}^{e}}+\frac{\Gamma_{1}}{2} p_{B_{-}^{o}}+\frac{2 \Gamma_{m}+\Gamma_{2}}{4} \xi, \\
\frac{d}{d t} p_{B_{-}^{o}} & =-\frac{2 \Gamma_{m}+\Gamma_{1}+\Gamma_{2}}{2} p_{B_{-}^{o}}+\frac{\Gamma_{1}}{2} p_{B_{+}^{e}}+\frac{\Gamma_{2}}{4} \xi, \\
\frac{d}{d t} \xi & =-\frac{\Gamma_{m}+\Gamma_{2}}{2} \xi+\frac{\Gamma_{2}}{2} p_{B_{+}^{e}}+\frac{2 \Gamma_{m}+\Gamma_{2}}{2} p_{B_{-}^{o} .}
\end{aligned}
$$

Here, we have defined

$$
\begin{aligned}
\Gamma_{m} & =\frac{r_{\text {parity }}}{T_{d}}, \\
\Gamma_{1} & =\frac{1}{2 T_{1}^{A}}+\frac{1}{2 T_{1}^{B}}, \\
\Gamma_{2} & =\frac{r_{\text {dephasing }}}{T_{d}}+\frac{1}{2 T_{1}^{A}}+\frac{1}{2 T_{1}^{B}}+\frac{1}{T_{\phi}^{A}}+\frac{1}{T_{\phi}^{B}} .
\end{aligned}
$$

Solving for the steady state gives

$$
\begin{aligned}
p_{B_{+}^{e}} & =\frac{(1+\tilde{\delta}) \Gamma_{1} / \Gamma_{2}+(\tilde{\delta})^{2}}{4(1+\tilde{\delta}) \Gamma_{1} / \Gamma_{2}+(1+\tilde{\delta})^{2}}, \\
\text { with } \quad \tilde{\delta} & =\frac{2 r_{\text {parity }}}{T_{d} \Gamma_{2}}+1 .
\end{aligned}
$$

From this expression, the optimum value of $\alpha$ can easily be computed numerically. We have simulated the actual system dynamics, i.e., adding qubit decoherence (7) to (3), for different values of $\eta$ and $T_{d} / T_{1}$ (we have taken $T_{1}^{A}=T_{1}^{B}=T_{1}$ and $\left.T_{\phi}^{A, B}=\infty\right)$. For $\alpha$ we have taken the value that maximizes Eq. (8), as well as a few values close to it in order to confirm that we hit close to the actual optimum fidelity. For each set of parameters, we have performed 5000 Monte Carlo simulations in order to estimate the achieved fidelity. Figure 6 shows these simulation results. The value of $\alpha$ obtained by maximizing Eq. (8) appears to indeed be close to optimal. While the above simple scheme is certainly not the optimal feedback strategy, the simulation results on Fig. 6 are indicative of the minimal entanglement achievable with our remote parity measurement. They show that if, e.g., 1000 measurements can be performed during a qubit lifetime (comparing $100 \mu$ s lifetime to $100 \mathrm{~ns}$ duration of the most demanding measurement operation) and transmission fidelity reaches about $85 \%$, then the steady-state entanglement fidelity that is stabilized for all times can be pushed up to $98 \%$.

\section{EXPERIMENTAL CONSIDERATIONS}

All the required operations for the above proposal have been individually implemented within the framework of quantum superconducting circuits, where the cavity-qubit setting is the standard minimal building block. The strong dispersive coupling of a transmon qubit to a high- $Q$ cavity mode [32] provides universal controllability of the state of the quantum harmonic oscillator modeling the cavity mode [33,34]. This controllability has been experimentally illustrated with circuit QED setups [24,35]. Such a coupling enables us to prepare the probe field in a cat state and to perform the CNOT gates $U_{A}$ and $U_{B}$ of Eq. (1) between the qubits and associated intracavity fields. Recent experiments realizing a variable coupling between cavity modes and a transmission line [21-23]

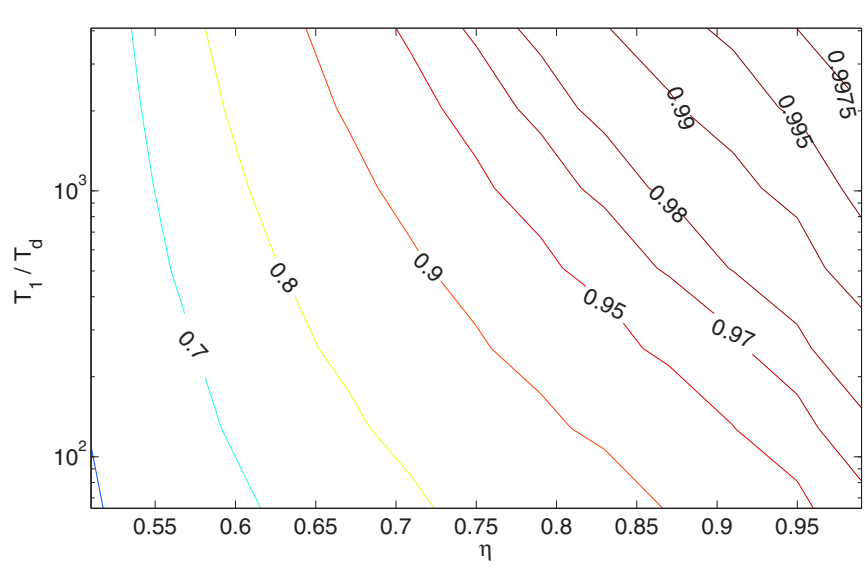

FIG. 6. Fidelities to the Bell state $\left|B_{+}^{e}\right\rangle=(|00\rangle+|11\rangle) / \sqrt{2}$ obtained by our remote entanglement feedback stabilization scheme in the presence of individual qubit decay (7). Here, we have assumed $T_{1}^{A}=T_{1}^{B}=T_{1}$ and $T_{\phi}^{A, B}=\infty$. For each value of $\eta$ (transmission fidelity of the meter quantum channel for the remote parity measurement) and of $T_{1} / T_{d}$ (qubit characteristic lifetime expressed in number of measurement iterations), we have estimated the optimal value of $\alpha$ by maximizing Eq. (8). We have then run 5000 Monte Carlo simulations with values of $\alpha$ up to two times larger or smaller than this estimated optimum and selected the best result. The latter turned out to be close to the computed $\alpha$ by optimizing Eq. (8). To estimate steady-state fidelity, we have averaged the fidelity obtained between $t=3 T_{1}$ and $t=6 T_{1}$, when starting at $t=0$ from $\left|B_{+}^{e}\right\rangle$. We have checked that this initial state does not affect the steady state; but starting close to the expected steady state allows us to minimize the transient effects.

provide the possibility of catching the propagating microwave field, performing the required gate between the qubit and the cavity field, and finally releasing back the cavity photons [36]. Finally, while the measurement of photon-number parity has also been realized in a similar setup [37], one can further simplify the protocol by letting $U_{B}$ map the intracavity field to coherent states $| \pm \sqrt{\eta} \alpha\rangle$ instead of the cat states $\left|\mathcal{C}_{\sqrt{\eta} \alpha}^{ \pm}\right\rangle$. This would allow us to replace a photon-number parity measurement by a simple homodyne detection of the released field using a parametric amplifier [20]. A single measurement duration in such an experimental realization depends mainly on the time required to perform the operations $U_{A}$ and $U_{B}$. As explained in Refs. [33,34] this gate time is roughly the inverse of the dispersive coupling strength. Recent experiments where this coupling strength is more than three orders of magnitude larger than both the qubit and the cavity decay rates $[24,35,38]$ indicate that high entanglement fidelities should be achievable whenever a transmission efficiency of more than $70 \%$ is reached.

\section{CONCLUSION}

We have shown that it is possible to perform a near DP-QND measurement of the parity of two distant qubits despite an important loss through the transmission channel between them. By preparing the probe field in a quantum superposition of two coherent states with opposite phases, we avoid backaction of the probe losses on the target qubits' state inside any 
parity subspace. Indeed, such losses mainly decrease the measurement strength but barely affect its DP-QND character. Therefore, even with an inefficient transmission channel, by repeating the measurement many times one efficiently projects the joint qubits state onto a definite-parity subspace. This enables not only to deterministically and hardware-efficiently prepare an entangled state of two distant qubits but, combined with a quantum feedback strategy, it also protects such a state against decoherence channels and systematic errors. The operations required to perform this loss-tolerant parity measurement are within the reach of state-of-the-art experiments. Their implementation will lead to an important step forward for implementing quantum teleportation protocols in a loss-tolerant way, and more particularly towards the modular architecture solution for large-scale quantum information processors [4].

\section{ACKNOWLEDGMENT}

The authors thank the Agence Nationale de la Recherche for financial support under Grant ANR-14-CE26-0018.

\section{APPENDIX A: NECESSARY CONDITION FOR LOSS-TOLERANT DP-QND MEASUREMENTS}

We prove that it is impossible to design the measurement setup of Fig. 1(a) in order to obtain a DP-QND parity measurement under arbitrary channel noise, i.e., when $E_{k}$ in Fig. 1(b) can be arbitrary.

(S0) Note that any unconditioned local unitaries on the qubits along the measurement chain can be merged into $U_{A}$ and $U_{B}$. Indeed, even if physically we apply such $\tilde{U}_{A}$ to qubit $A$ after $U_{B}$, this $\tilde{U}_{A}$ commutes with $U_{B}$ since it acts as identity on both $\left|q_{B}\right\rangle$ and $|\psi\rangle_{p}$. We will thus assume without loss of generality a setting with just two arbitrary unitaries $U_{A}$ and $U_{B}$, which make the arbitrary probe interact respectively with $\left|q_{A}\right\rangle$ and $\left|q_{B}\right\rangle$.

(S1) To keep invariant the even-parity states $|00\rangle_{A, B}$ and $|11\rangle_{A, B}$, we have to take $U_{A}$ such that $U_{A}|0\rangle_{A}\left|\psi_{p}\right\rangle=|0\rangle_{A}\left|\phi_{0}\right\rangle_{p}$ and $U_{A}|1\rangle_{A}\left|\psi_{p}\right\rangle=|1\rangle_{A}\left|\phi_{1}\right\rangle_{p}$ for some $\left|\phi_{0}\right\rangle$, $\left|\phi_{1}\right\rangle$. Indeed consider a contrario that, e.g., an initial $|0\rangle_{A}$ was mapped by $U_{A}$ into a state involving $|1\rangle_{A}$. Then, since no further action is applied on qubit $A$, at the end of the nominal measurement chain there would unavoidably be a nonzero probability to end up in a state involving this $|1\rangle_{A}$, which would contradict the DP-QND objective for an initial state $|00\rangle_{A, B}$.

(S2) If we chose $\left|\phi_{1}\right\rangle=e^{i \theta}\left|\phi_{0}\right\rangle$ for some $\theta$, then we could rewrite

$$
U_{A}\left|q_{A}\right\rangle\left|\psi_{p}\right\rangle=\left(Z_{\theta}\left|q_{A}\right\rangle\right)\left|\phi_{0}\right\rangle_{p}
$$

with $Z_{\theta}$ acting only on qubit $A$ and defined by $Z_{\theta}|0\rangle_{A}=$ $|0\rangle_{A}, Z_{\theta}|1\rangle_{A}=e^{i \theta}|1\rangle_{A}$. This clarifies that the probe state $\left|\phi_{0}\right\rangle_{p}$ would carry no information about the state of qubit $A$, hence we can get no parity information by later measuring the probe system at $B$.

(S3a) On the other hand, if we have $\left|\phi_{1}\right\rangle \neq e^{i \theta}\left|\phi_{0}\right\rangle$, then it is always possible to write a noise channel which keeps $\left|\phi_{0}\right\rangle$ in place but, conditionally on the unknown state $\left|q_{\text {env }}\right\rangle$ of some environment variable, moves $\left|\phi_{1}\right\rangle$. In other words, referring to Fig. 1(b),

$$
E_{1}\left|\phi_{0}\right\rangle_{p}=\left|\phi_{0}\right\rangle_{p} \quad \text { and } \quad E_{1}\left|\phi_{1}\right\rangle_{p}=\left|\phi_{2}\right\rangle_{p} \neq\left|\phi_{1}\right\rangle_{p} .
$$

(S3b) Consider now what happens to the initial even-parity state $(|00\rangle+|11\rangle)_{A, B} / \sqrt{2}$. After $U_{A}$ has been applied, the qubits are entangled with the probe in the state $\left(|00\rangle_{A, B}\left|\phi_{0}\right\rangle_{p}+\right.$ $\left.|11\rangle_{A, B}\left|\phi_{1}\right\rangle_{p}\right) / \sqrt{2}$. To have a DP-QND measurement, the remaining action $U_{B}$ has to disentangle $(|00\rangle+|11\rangle)_{A, B} / \sqrt{2}$ from the probe before detection. In the case where $E_{1}$ is not applied (no channel loss), we thus need

$U_{B}|0\rangle_{B}\left|\phi_{0}\right\rangle_{p}=|0\rangle_{B}\left|\phi_{b}\right\rangle_{p} \quad$ and $\quad U_{B}|1\rangle_{B}\left|\phi_{1}\right\rangle_{p}=|1\rangle_{B}\left|\phi_{b}\right\rangle_{p}$

for some $\left|\phi_{b}\right\rangle$. In the case where $E_{1}$ in contrast is applied by the lossy channel, we need

$U_{B}|0\rangle_{B}\left|\phi_{0}\right\rangle_{p}=|0\rangle_{B}\left|\phi_{a}\right\rangle_{p} \quad$ and $\quad U_{B}|1\rangle_{B}\left|\phi_{2}\right\rangle_{p}=|1\rangle_{B}\left|\phi_{a}\right\rangle_{p}$

for some $\left|\phi_{a}\right\rangle$. For the experiment we must select a unique $U_{B}$, without knowing the environment state $\left|q_{\text {env }}\right\rangle$ i.e., without knowing whether $E_{1}$ was applied or not. The left parts of Eqs. (A1) and (A2) thus impose $\left|\phi_{b}\right\rangle=\left|\phi_{a}\right\rangle$. But then the second parts of Eqs. (A1) and (A2) lead to a contradiction, as they require the unitary $U_{B}$ to map two different initial states $|1\rangle_{B}\left|\phi_{1}\right\rangle_{p} \neq|1\rangle_{B}\left|\phi_{2}\right\rangle_{p}$ onto the same final state.

This shows the impossibility for the measurement to be DP-QND with respect to the "conditional $E_{1}$ " channel noise. We have taken care to keep our construction fully general, such that we can conclude the following: Whatever our design for $\left|\psi_{p}\right\rangle, U_{A}$, and $U_{B}$, we will obtain a measurement setup either which gains no parity information, or for which some particular noise actions $E_{1}$ can destroy the DP-QND character and perturb definite-parity states.

For example, in our proposed construction with CNOT gates:

(1) For S1 we have $\left|\phi_{0}\right\rangle=|0\rangle,\left|\phi_{1}\right\rangle=|1\rangle$; we are obviously in the situation of S3a, not of S2.

(2) Regarding S3a, the phase-flip channel $\sigma_{z}$ for instance would keep $|0\rangle$ at $|0\rangle$ but move $|1\rangle$ to $-|1\rangle$. Then to save the situation $U_{B}$ would have to satisfy in particular $U_{B}|1\rangle_{B}|1\rangle_{p}=$ $-U_{B}|1\rangle_{B}|1\rangle_{p}$, which is not possible.

This example shows that a DP-QND parity measurement is not possible if the transmitted probe state can be subject to both bit flip and phase flip errors. In this paper, we discussed the case, motivated by realistic experimental conditions, where the transmission channel only subjects the probe to an unknown number of bit flips (probe's cat states). Consider indeed a situation where the transmission channel subjects the probe to an unknown number $n$ of unitary operations of the same type $U_{C}$, where $\left(U_{C}\right)^{N}$ equals identity for some integer $N$. Now let $U_{A}$ and $U_{B}$ apply conditional $V$ gates on the probe, where $V=\left(U_{C}\right)^{N / 2}$. Then all the operations $U_{A}, U_{B}, U_{C}$ commute with each other, and an initial probe state $\left|\psi_{0}\right\rangle_{p}$ gets mapped just before detection onto

$$
\left(U_{C}\right)^{N / 2}\left(U_{C}\right)^{N / 2} U_{C}^{n}\left|\psi_{0}\right\rangle_{p}=U_{C}^{n}\left|\psi_{0}\right\rangle_{p}
$$

if the target qubits have even parity, or onto

$$
\left(U_{C}\right)^{N / 2} U_{C}^{n}\left|\psi_{0}\right\rangle_{p}
$$


if they have odd parity, while the target qubits remain unaffected. Thus not knowing $n$ makes the final probe state uncertain, as with the bit flip, implying that detection results will not allow us to perfectly discriminate the parity. But the probe ends up in the same (unknown) state for all even-parity states of the target qubit; and it ends up in another same state for all odd-parity states of the target qubit. This ensures preservation of the DP-QND property under channel losses.

\section{APPENDIX B: KRAUS OPERATORS}

Here, we summarize the calculations that lead to the Kraus operators of Eq. (2). We start with an initial joint state of the two qubits, the probe field, and the ancillary mode modeling the transmission loss, given by

$$
\begin{aligned}
\left|\psi_{0}\right\rangle= & \left(c_{00}|00\rangle_{A, B}+c_{11}|11\rangle_{A, B}+c_{01}|01\rangle_{A, B}\right. \\
& \left.+c_{10}|10\rangle_{A, B}\right)\left|\mathcal{C}_{\alpha}^{+}\right\rangle_{p}|0\rangle_{\mathrm{env}} .
\end{aligned}
$$

Following the definition of the unitary operators $U_{A}, U_{B}$, and $U_{\mathrm{BS}}^{\eta}$ in Eq. (1), just before performing the photon-number parity measurements of the probe and ancillary fields, this joint state has evolved to

$$
\begin{aligned}
|\psi\rangle= & \frac{c_{00}}{2 \mathcal{N}_{\alpha}^{+}}|00\rangle_{A, B}\left[\left|\mathcal{C}_{\sqrt{\eta} \alpha}^{+}\right\rangle_{p}\left|\mathcal{C}_{\sqrt{1-\eta} \alpha}^{+}\right\rangle_{\text {env }} \mathcal{N}_{\sqrt{\eta} \alpha}^{+} \mathcal{N}_{\sqrt{1-\eta} \alpha}^{+}\right. \\
& \left.+\left|\mathcal{C}_{\sqrt{\eta} \alpha}^{-}\right\rangle_{p}\left|\mathcal{C}_{\sqrt{1-\eta} \alpha}^{-}\right\rangle_{\text {env }} \mathcal{N}_{\sqrt{\eta} \alpha}^{-} \mathcal{N}_{\sqrt{1-\eta} \alpha}^{-}\right] \\
& +\frac{c_{11}}{2 \mathcal{N}_{\alpha}^{-}}|11\rangle_{A, B}\left[\left|\mathcal{C}_{\sqrt{\eta} \alpha}^{+}\right\rangle_{p}\left|\mathcal{C}_{\sqrt{1-\eta} \alpha}^{+}\right\rangle_{\text {env }} \mathcal{N}_{\sqrt{\eta} \alpha}^{-} \mathcal{N}_{\sqrt{1-\eta} \alpha}^{+}\right. \\
& \left.+\left|\mathcal{C}_{\sqrt{\eta} \alpha}^{-}\right\rangle_{p}\left|\mathcal{C}_{\sqrt{1-\eta} \alpha}^{-}\right\rangle_{\text {env }} \mathcal{N}_{\sqrt{\eta} \alpha}^{+} \mathcal{N}_{\sqrt{1-\eta} \alpha}^{-}\right] \\
& +\frac{c_{01}}{2 \mathcal{N}_{\alpha}^{+}}|01\rangle_{A, B}\left[\left|\mathcal{C}_{\sqrt{\eta} \alpha}^{+}\right\rangle_{p}\left|\mathcal{C}_{\sqrt{1-\eta} \alpha}^{-}\right\rangle_{\text {env }} \mathcal{N}_{\sqrt{\eta} \alpha}^{-} \mathcal{N}_{\sqrt{1-\eta} \alpha}^{-}\right. \\
& \left.+\left|\mathcal{C}_{\sqrt{\eta} \alpha}^{-}\right\rangle_{p}\left|\mathcal{C}_{\sqrt{1-\eta} \alpha}^{+}\right\rangle_{\text {env }} \mathcal{N}_{\sqrt{\eta} \alpha}^{+} \mathcal{N}_{\sqrt{1-\eta} \alpha}^{+}\right] \\
& +\frac{c_{10}}{2 \mathcal{N}_{\alpha}^{-}}|10\rangle_{A, B}\left[\left|\mathcal{C}_{\sqrt{\eta} \alpha}^{+}\right\rangle_{p}\left|\mathcal{C}_{\sqrt{1-\eta} \alpha}^{-}\right\rangle_{\text {env }} \mathcal{N}_{\sqrt{\eta} \alpha}^{+} \mathcal{N}_{\sqrt{1-\eta} \alpha}^{-}\right. \\
& \left.+\left|\mathcal{C}_{\sqrt{\eta} \alpha}^{-}\right\rangle_{p}\left|\mathcal{C}_{\sqrt{1-\eta} \alpha}^{+}\right\rangle_{\text {env }} \mathcal{N}_{\sqrt{\eta} \alpha}^{-} \mathcal{N}_{\sqrt{1-\eta} \alpha}^{+}\right] .
\end{aligned}
$$

Here, we have used the fact that

$$
| \pm \beta\rangle=\frac{\mathcal{N}_{\beta}^{+}}{2}\left|\mathcal{C}_{\beta}^{+}\right\rangle \pm \frac{\mathcal{N}_{\beta}^{-}}{2}\left|\mathcal{C}_{\beta}^{-}\right\rangle
$$

for $\beta=\sqrt{\eta} \alpha$ and $\beta=\sqrt{1-\eta} \alpha$. Detecting the probe and the ancillary fields both in even parity leads to applying the following projection operator as the measurement backaction:

$$
\begin{aligned}
\Pi_{+,+} & =\operatorname{Id}_{A, B} \otimes \Pi_{p}^{\text {even }} \otimes \Pi_{\text {env }}^{\text {even }} \\
& =\operatorname{Id}_{A, B} \otimes\left(\sum _ { k = 0 } ^ { \infty } | 2 k \rangle \langle 2 k | _ { p } ) \otimes \left(\sum_{k=0}^{\infty}|2 k\rangle\left\langle\left. 2 k\right|_{\text {env }}\right),\right.\right.
\end{aligned}
$$

with Id being the identity map. Since $\left|\mathcal{C}_{\beta}^{+}\right\rangle$has even parity and $\left|\mathcal{C}_{\beta}^{-}\right\rangle$has odd parity, the projected wave function can be simply read off the above expression,

$$
\begin{aligned}
\Pi_{+,+}|\psi\rangle= & \frac{\mathcal{N}_{\sqrt{1-\eta} \alpha}^{+}}{2}\left(c_{00} \frac{\mathcal{N}_{\sqrt{\eta} \alpha}^{+}}{\mathcal{N}_{\alpha}^{+}}|00\rangle+c_{11} \frac{\mathcal{N}_{\sqrt{\eta} \alpha}^{-}}{\mathcal{N}_{\alpha}^{-}}|11\rangle\right)_{A, B} \\
& \left|\mathcal{C}_{\sqrt{\eta} \alpha}^{+}\right\rangle_{p}\left|\mathcal{C}_{\sqrt{1-\eta} \alpha}^{+}\right\rangle_{\text {env }} .
\end{aligned}
$$

In a similar way, we have the following projected wave functions for other measurement outcomes:

$$
\begin{aligned}
\Pi_{+,-}|\psi\rangle= & \frac{\mathcal{N}_{\sqrt{1-\eta} \alpha}^{-}}{2}\left(c_{01} \frac{\mathcal{N}_{\sqrt{\eta} \alpha}^{-}}{\mathcal{N}_{\alpha}^{+}}|01\rangle+c_{10} \frac{\mathcal{N}_{\sqrt{\eta} \alpha}^{+}}{\mathcal{N}_{\alpha}^{-}}|10\rangle\right)_{A, B} \\
& \left|\mathcal{C}_{\sqrt{\eta} \alpha}^{+}\right\rangle_{p}\left|\mathcal{C}_{\sqrt{1-\eta} \alpha}^{-}\right\rangle_{\text {env }}, \\
\Pi_{-,+}|\psi\rangle= & \frac{\mathcal{N}_{\sqrt{1-\eta} \alpha}^{+}}{2}\left(c_{01} \frac{\mathcal{N}_{\sqrt{\eta} \alpha}^{+}}{\mathcal{N}_{\alpha}^{+}}|01\rangle+c_{10} \frac{\mathcal{N}_{\sqrt{\eta} \alpha}^{-}}{\mathcal{N}_{\alpha}^{-}}|10\rangle\right)_{A, B} \\
& \left|\mathcal{C}_{\sqrt{\eta} \alpha}^{-}\right\rangle_{p}\left|\mathcal{C}_{\sqrt{1-\eta} \alpha}^{+}\right\rangle_{\text {env }} \\
\Pi_{-,-}|\psi\rangle= & \frac{\mathcal{N}_{\sqrt{1-\eta} \alpha}^{-}}{2}\left(c_{00} \frac{\mathcal{N}_{\sqrt{\eta} \alpha}^{-}}{\mathcal{N}_{\alpha}^{+}}|00\rangle+c_{11} \frac{\mathcal{N}_{\sqrt{\eta} \alpha}^{+}}{\mathcal{N}_{\alpha}^{-}}|11\rangle\right)_{A, B} \\
& \left|\mathcal{C}_{\sqrt{\eta} \alpha}^{-}\right\rangle_{p}\left|\mathcal{C}_{\sqrt{1-\eta} \alpha}^{-}\right\rangle_{\text {env }} .
\end{aligned}
$$

This corresponds to the Kraus operators as defined by Eq. (2).
[1] J. S. Bell, Physics 1, 195 (1964).

[2] A. Aspect, P. Grangier, and G. Roger, Phys. Rev. Lett. 47, 460 (1981).

[3] C. H. Bennett, G. Brassard, C. Crépeau, R. Jozsa, A. Peres, and W. K. Wootters, Phys. Rev. Lett. 70, 1895 (1993).

[4] M. Devoret and R. Schoelkopf, Science 339, 1169 (2013).

[5] C. Monroe, R. Raussendorf, A. Ruthven, K. R. Brown, P. Maunz, L.-M. Duan, and J. Kim, Phys. Rev. A 89, 022317 (2014).

[6] H.-J. Briegel, W. Dür, J. I. Cirac, and P. Zoller, Phys. Rev. Lett. 81, 5932 (1998).

[7] L.-M. Duan, M. Lukin, J. Cirac, and P. Zoller, Nature (London) 414, 413 (2001).

[8] N. Roch, M. E. Schwartz, F. Motzoi, C. Macklin, R. Vijay, A. W. Eddins, A. N. Korotkov, K. B. Whaley, M. Sarovar, and I. Siddiqi, Phys. Rev. Lett. 112, 170501 (2014).

[9] C. Cabrillo, J. I. Cirac, P. García-Fernández, and P. Zoller, Phys. Rev. A 59, 1025 (1999).
[10] S. D. Barrett and P. Kok, Phys. Rev. A 71, 060310(R) (2005).

[11] L. Jiang, J. Taylor, A. Soerensen, and M. Lukin, Int. J. Quantum Inform. 8, 93 (2010).

[12] T. Ladd, P. van Loock, K. Nemoto, W. Munro, and Y. Yamamoto, New J. Phys. 8, 184 (2006).

[13] A. Campbell, Phys. Rev. A 76, 040302 (2007).

[14] C. Chou, H. de Riedmatten, D. Felinto, S. Polyakov, S. van Enk, and H. Kimble, Nature (London) 438, 828 (2005).

[15] D. Moehring, P. Maunz, S. Olmschenk, K. Younge, D. Matsukevich, L.-M. Duan, and C. Monroe, Nature (London) 449, 68 (2007).

[16] A. Ourjoumtsev, F. Ferreyrol, R. Tualle-Brouri, and P. Grangier, Nat. Phys. 5, 189 (2009).

[17] J. Hofmann, M. Krug, N. Ortegel, L. Gérard, M. Weber, W. Rosenfeld, and H. Weinfurter, Science 337, 72 (2012).

[18] H. Bernien, B. Hensen, W. Pfaff, G. Koolstra, M. Blok, L. Robledo, T. Taminiau, M. Markham, D. Twitchen, L. Childress, and R. Hanson, Nature (London) 497, 86 (2013). 
[19] A. Narla, S. Shankar, M. Hatridge, Z. Leghtas, K. M. Sliwa, E. Zalys-Geller, S. O. Mundhada, W. Pfaff, L. Frunzio, R. J. Schoelkopf, and M. H. Devoret, Phys. Rev. X 6, 031036 (2016).

[20] R. Vijay, M. H. Devoret, and I. Siddiqi, Rev. Sci. Instrum. 80, 111101 (2009).

[21] Y. Yin, Y. Chen, D. Sank, P. J. J. O’Malley, T. C. White, R. Barends, J. Kelly, E. Lucero, M. Mariantoni, A. Megrant, C. Neill, A. Vainsencher, J. Wenner, A. N. Korotkov, A. N. Cleland, and J. M. Martinis, Phys. Rev. Lett. 110, 107001 (2013).

[22] J. Wenner, Y. Yin, Y. Chen, R. Barends, B. Chiaro, E. Jeffrey, J. Kelly, A. Megrant, J. Y. Mutus, C. Neill, P. J. J. O'Malley, P. Roushan, D. Sank, A. Vainsencher, T. C. White, A. N. Korotkov, A. N. Cleland, and J. M. Martinis, Phys. Rev. Lett. 112, 210501 (2014).

[23] E. Flurin, N. Roch, J. D. Pillet, F. Mallet, and B. Huard, Phys. Rev. Lett. 114, 090503 (2015).

[24] R. W. Heeres, B. Vlastakis, E. Holland, S. Krastanov, V. V. Albert, L. Frunzio, L. Jiang, and R. J. Schoelkopf, Phys. Rev. Lett. 115, 137002 (2015).

[25] R. W. Heeres, P. Reinhold, N. Ofek, L. Frunzio, L. Jiang, M. H. Devoret, and R. J. Schoelkopf, arXiv:1608.02430.

[26] Z. Leghtas, G. Kirchmair, B. Vlastakis, R. J. Schoelkopf, M. H. Devoret, and M. Mirrahimi, Phys. Rev. Lett. 111, 120501 (2013).

[27] A. Wallraff, D. Schuster, A. Blais, L. Frunzio, R. Huang, J. Majer, S. Kumar, S. Girvin, and R. J. Schoelkopf, Nature (London) 431, 162 (2004).

[28] K. Lalumière and A. Blais, Physics 7, 45 (2014).
[29] M. Nielsen and I. Chuang, Quantum Computation and Quantum Information (Cambridge University Press, Cambridge, UK, 2000).

[30] M. Mirrahimi, Z. Leghtas, V. V. Albert, S. Touzard, R. J. Schoelkopf, L. Jiang, and M. H. Devoret, New J. Phys. 16, 045014 (2014).

[31] P. Rouchon, IEEE Trans. Autom. Control 56, 2743 (2011).

[32] D. Schuster, A. Houck, J. Schreier, A. Wallraff, J. Gambetta, A. Blais, L. Frunzio, J. Majer, B. Johnson, M. H. Devoret, S. Girvin, and R. J. Schoelkopf, Nature (London) 445, 515 (2007).

[33] Z. Leghtas, G. Kirchmair, B. Vlastakis, M. H. Devoret, R. J. Schoelkopf, and M. Mirrahimi, Phys. Rev. A 87, 042315 (2013).

[34] S. Krastanov, V. V. Albert, C. Shen, C.-L. Zou, R. W. Heeres, B. Vlastakis, R. J. Schoelkopf, and L. Jiang, Phys. Rev. A 92, 040303(R) (2015).

[35] B. Vlastakis, G. Kirchmair, Z. Leghtas, S. Nigg, L. Frunzio, S. Girvin, M. Mirrahimi, M. H. Devoret, and R. J. Schoelkopf, Science 342, 607 (2013).

[36] W. Pfaff, C. J. Axline, L. D. Burkhart, U. Vool, P. Reinhold, L. Frunzio, L. Jiang, M. H. Devoret, and R. J. Schoelkopf, arXiv:1612.05238.

[37] L. Sun, A. Petrenko, Z. Leghtas, B. Vlastakis, G. Kirchmair, K. Sliwa, A. Narla, M. Hatridge, S. Shankar, J. Blumoff, L. Frunzio, M. Mirrahimi, M. H. Devoret, and R. J. Schoelkopf, Nature (London) 511, 444 (2014).

[38] H. Paik, D. I. Schuster, L. S. Bishop, G. Kirchmair, G. Catelani, A. P. Sears, B. R. Johnson, M. J. Reagor, L. Frunzio, L. I. Glazman, S. M. Girvin, M. H. Devoret, and R. J. Schoelkopf, Phys. Rev. Lett. 107, 240501 (2011). 